\title{
Acoustic antialiasing filter having one resistive element for digitized microphone
}

\author{
Hikaru Date* and Kenzo Miura** \\ *Kyushu Institute of Design, \\ 226, Shiobaru, Minami-ku, Fukuoka, 815 Japan \\ **Matsushita Communication Industry Co. Ltd., \\ 4-3-1, Tsunashima, Kohoku-ku, Yokohama, 223 Japan
}

(Received 4 September 1981)

\begin{abstract}
The synthetic method of electrical passive networks is successfully applied to the development of an acoustic antialiasing filter for an electret condenser microphone. The Butterworth type lowpass filter of order nine is realized as a tandem connection of an acoustic filter of order five and an electronic filter of order four which is imbeded in the microphone amplifier. The merit of this synthetic design is that the filter can be so realized with only one acoustic resistance that makes the adjustment at the production easy.
\end{abstract}

PACS number: 43. 88, $-\mathrm{p}$

\section{INTRODUCTION}

In 1979, Flanagan of Bell Laboratories first introduced the necessity, the design method and examples of acoustic antialiasing filter for so-called "Digital Voice."1) Here, we are going to describe another design method using only one acoustic resistance and an example of an acoustic filter for digitized microphone* recently developed. ${ }^{2-4)}$

The object of this paper is to introduce a precise design method after the synthetic theory of linear passive networks and a slight modification process by computer aided design method for realization of acoustic antialiasing filter.

\section{PHYSICAL CONSTRAINTS}

We usually face the following physical constraints at the realization of acoustic filter to be used by

* The term "digitized microphone" means a microphone with digitized output. We realized a digitized microphone ${ }^{2)}$ by tandem connection of four elements: an acoustic antialiasing filter, ${ }^{3)}$ an electret condenser microphone, a microphone amplifier and a 14 to 16 bits floating point A/D converter of parallel type.4) This paper describes the design method of the first element. attaching to a small condenser microphone:

(1) Acoustic circuit elements are usually lossless.

(2) The source impedance, that is, radiation load in terms of normalized acoustic impedance of the acoustic filter is small and reactive for wide range of frequencies.

(3) If a condenser microphone is used as a transducer, load impedance of the filter is very high except the region of neighbouring frequencies of resonance of condenser microphone.

\section{DESIGN PRINCIPLES}

Considering the constraints mentioned above, we adopt the following principles:

(1) Firstly, synthetic method should be used in order to determine the basic structure and element values of the filter. If the design becomes too complicated to apply synthetic method because of loading conditions at high frequencies, a computer aided design for slight modification of element values should be utilized.

(2) Physical constraints indicate the elements of the filter are essentially of high $Q$ type. In order to obtain flat amplitude-frequency characteristics below the cut-off frequency, we must use acoustic resistance 
at certain point of the circuit. The number of resistances, however, should be limitted to only one so that we are able to reduce the difficulties of adjustment at the production.

\section{SYNTHETIC DESIGN METHOD ${ }^{5)}$}

Figure 1 depicts the general structure of the acoustic filter which satisfies the design principle. A resistance $R_{1}$ is added artificially. The opencircuit transmission coefficient $N_{0}$ is given by the following equation:

$$
N_{0}=V_{0} / V_{1}=C R_{1}+A=\left(R_{1}+Z_{11}\right) / Z_{12},
$$

where $C$ and $A$ are elements of $F$-matrix, and $Z_{11}$ and $Z_{12}$ are elements of $Z$-matrix of the lossless network. Hereafter, we regard $Z_{11}$ and $Z_{12}$ as the normalized impedances by $R_{1}$. Then we get

$$
N_{0}^{-1}=\frac{Z_{12}}{1+Z_{11}} \text {. }
$$

As $N_{0}^{-1}$ is a rational function of $s$, it is generally represented by

$$
N_{0}^{-1}=\frac{g(s)}{q(s)}=\frac{m+n}{m_{2}+n_{2}},
$$

where $m$ and $n$ are even and odd components of numerator polynomial $g(s)$ respectively, and $m_{2}$ and $n_{2}$ are even and odd components of denominator polynomial $q(s)$ respectively.

Because $Z_{11}$ and $Z_{12}$ are restricted to lossless, they must be ratios of even and odd polynomials. Therefore, $N_{0}^{-1}$ should be

$$
\begin{aligned}
\text { either } & N_{0}^{-1}=\frac{m}{m_{2}+n_{2}} \\
\text { or } & N_{0}^{-1}=\frac{n}{m_{2}+n_{2}}
\end{aligned}
$$

instead of the general expression of Eq. (3). Here, of course, $m_{2}+n_{2}$ is equal to the denominator

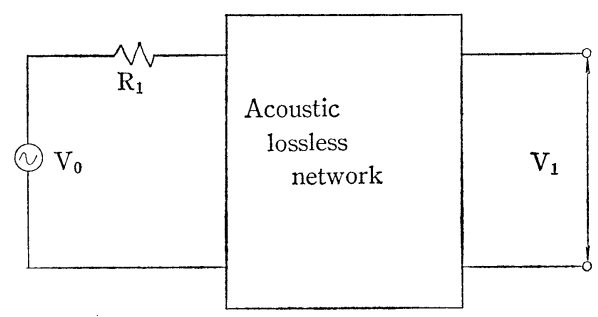

Fig. 1 Lossless network with lossy voltage source and infinite load impedance. polynomial of the driving point impedance of the lossless acoustic network.

We assume $\left|N_{0}^{-1}\right|^{2}$ is prescribed as a function of frequency. Returning to Eq. (3), we get

$$
\left|N_{0}\right|^{-2}=\frac{g(s) g(-s)}{q(s) q(-s)}=\left[\frac{m^{2}-n^{2}}{m_{2}^{2}-n_{2}^{2}}\right]_{s=j \omega} .
$$

Then, $\left|N_{0}\right|^{-2}$ can be expanded as follows:

$$
\left|N_{0}\right|^{-2}=\frac{A_{0}+A_{1} \omega^{2} \cdots+A_{p} \omega^{2 p}}{B_{0}+B_{1} \omega^{2} \cdots+B_{p} \omega^{2 p}}=\frac{A\left(\omega^{2}\right)}{B\left(\omega^{2}\right)} .
$$

Comparing Eqs. (2) and (4), we conclude that

$$
\begin{aligned}
& \text { either } \quad Z_{12}=m / n_{2} \text { and } Z_{11}=m_{2} / n_{2} \quad \text { (A) } \\
& \text { or } Z_{12}=n / m_{2} \text { and } Z_{11}=n_{2} / m_{2} \quad \text { (B) }
\end{aligned}
$$

should be held.

Combining Eqs. (5) (7), we obtain

$$
\begin{array}{ll}
\text { either } & A\left(-s^{2}\right)=A\left(\omega^{2}\right)=m^{2} \quad \text { and } \\
& B\left(-s^{2}\right)=B\left(\omega^{2}\right)=m_{2}{ }^{2}-n_{2}{ }^{2} \\
\text { or } & -A\left(-s^{2}\right)=A\left(\omega^{2}\right)=n^{2} \text { and } \\
& B\left(-s^{2}\right)=B\left(\omega^{2}\right)=m_{2}{ }^{2}-n_{2}{ }^{2} .
\end{array}
$$

Because $m_{2}+n_{2}$ must be Hurwitz polynomial for the sake of physical realizability, its zeros are composed of zeros, which lie on the left half plane, of $B\left(-s^{2}\right)$.

From Eq. (8), $m$ and $n$ can be obtained as

$$
m=\sqrt{A\left(-s^{2}\right)} \text { and } n=\sqrt{-A\left(-s^{2}\right)},
$$

for both cases of (A) and (B). Using Eqs. (7) and (9), we can determine $Z_{11}$ and $Z_{12}$ as a rational function form. We can finally obtain the values of network elements by applying either Darlington or continued fraction expansion method to $Z_{11}$ and $Z_{12}$. In this section we briefly surveyed one of the well-known methods of transfer function synthesis for later explanations.

\section{DESIGN EXAMPLE}

Here we try to design a Butterworth low pass filter of order nine, which is realized by acoustic filter of order five and electronic active filter of order four in tandem. The electronic filter, of which auxiliary role is to suppress the effect of radial mode resonances of the acoustic system at higher frequency region than the cut-off frequency, is imbeded in the microphone amplifier.

Squared amplitude-frequency characteristics of Butterworth lowpass filter of order nine is express- 


\section{H. DATE and K. MIURA: ACOUSTIC ANTIALIASING FILTER}

ed as

$$
\left|N_{0}(j \omega)^{-1}\right|^{2}=1 /\left(1+\omega^{18}\right),
$$

and its poles which lie on the left half of $s$-plane are given by

$$
\begin{aligned}
s_{\nu}=e^{j(2 \pi / 18) \nu}, & \\
& \nu=5,6,7,8,9,10,11,12,13 .
\end{aligned}
$$

Of these poles, we select as the poles of the fifth order acoustic filter $s_{9}, s_{5,13}$ and $s_{6,12}$. Then we get the following denominator polynomial

$$
\begin{aligned}
m_{2}+n_{2}= & s^{5}+2.3473 s^{4}+3.6946 s^{3} \\
& +3.6946 s^{2}+2.3473 s+1,
\end{aligned}
$$

$$
Z_{11}=\frac{1}{0.4260 s+\frac{1}{1.1069 s}+\frac{1}{1.3525 s}+\frac{1}{2.7566 s}+\frac{1}{0.5688 s} .}
$$

Therefore, the elements $Z_{11}$ and $Z_{12}$ of open circuit impedance matrix is given by

$$
\begin{aligned}
& Z_{11}=\frac{2.3473 s^{4}+3.6946 s^{2}+1}{s^{5}+3.6946 s^{3}+2.3473 s} \\
& Z_{12}=\frac{1}{s^{5}+3.6946 s^{3}+2.3473 s} .
\end{aligned}
$$

Expanding $Z_{11}$ into the continued fraction form, we obtain

lossless acoustic filter indicates that a flat amplitude-frequency characteristics below $15 \mathrm{kHz}$ can be finally obtained if the cut-off frequency of the circuit shown in Fig. 2 is made $20 \mathrm{kHz}$. This charac-

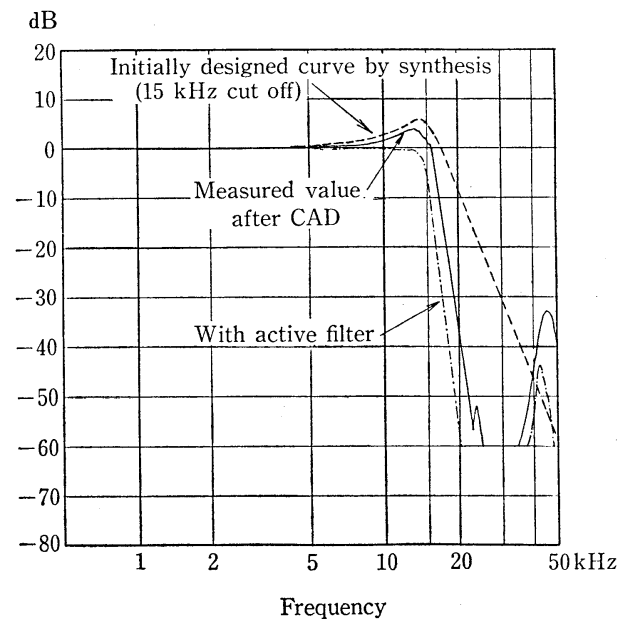

Fig. 3 Amplitude-frequency characteristics of the antialiasing filter.

Broken line: calculated curve for the circuit shown in Fig. 2, solid line: after computer aided design (measured value), chain line: over-all measured curve between acoustic filter input and electronic active filter output. 


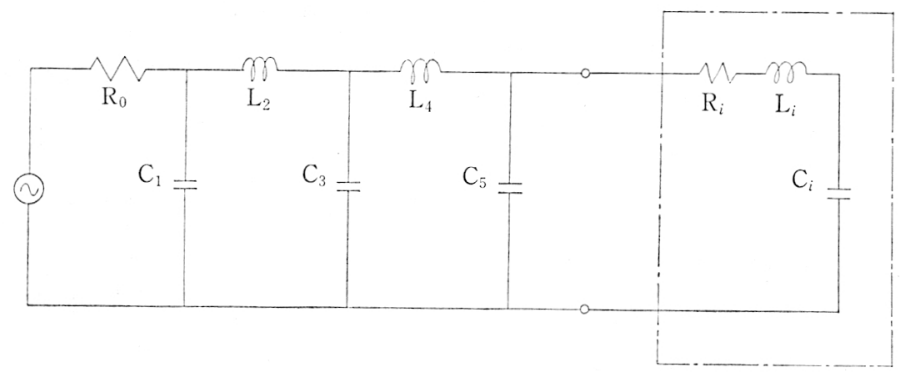

Fig. 4 Equivalent circuit diagram of the acoustic filter loaded by series-resonant circuit $\left(\mathrm{L}_{i}, \mathrm{C}_{i}\right.$ and $\mathrm{R}_{i}$ ) which represents microphone's resonance.

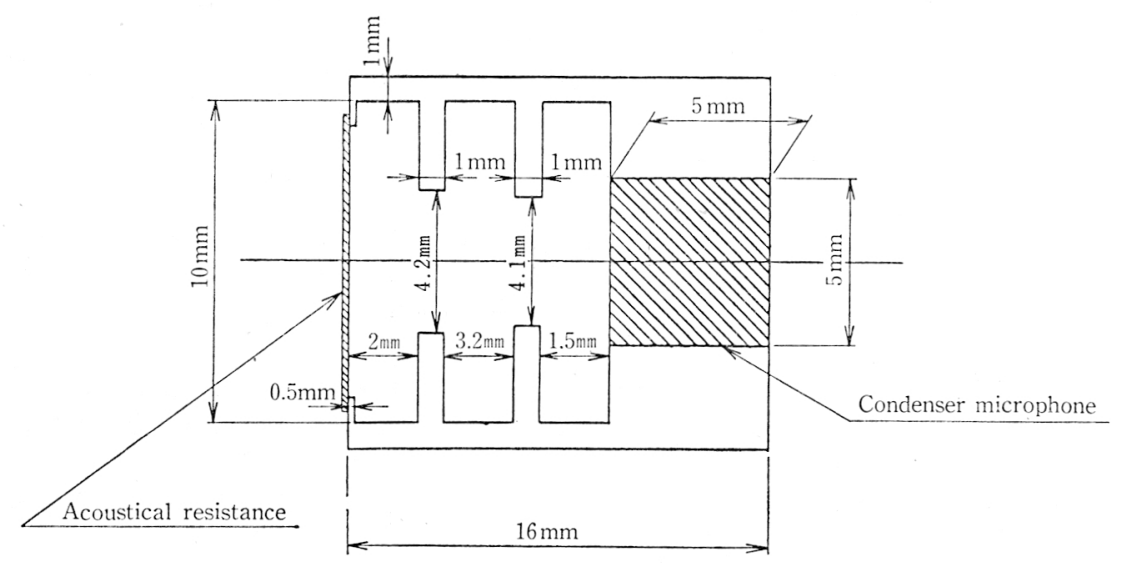

Fig. 5 Shape and size of the acoustic filter.

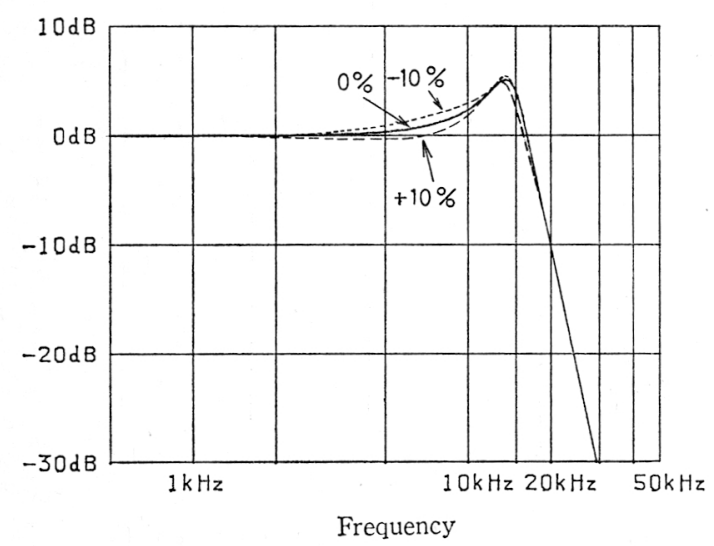

Fig. 6 The influence of the acoustic resistance inaccuracy on the acoustic filter response.

teristics is indicated by solid line in Fig. 3. The chain line is the measured value of the over-all

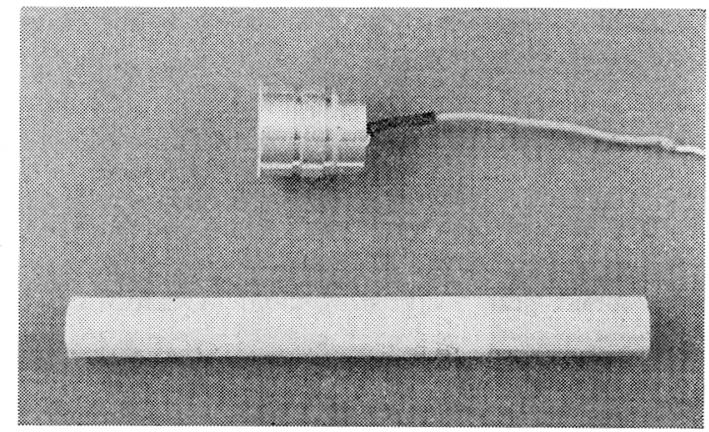

Fig. 7 Photograph of the acoustic filter with electret condenser microphone.

amplitude-frequency characteristics of the acoustic filter and the electronic filter in tandem connection. A flat amplitude-frequency characteristics within passband under about $15 \mathrm{kHz}$ and more than 40 $\mathrm{dB}$ attenuation in stop band are realized. 


\section{H. DATE and K. MIURA: ACOUSTIC ANTIALIASING FILTER}

Figure 5 shows the drawing of the acoustic filter structure in which the acoustic resistance 87.7 (cgs) is realized by thin nylon cloth. Figure 6 depicts the influence of the acoustic resistance inaccuracy lying $\pm 10 \%$ in commercially available materials on the calculated frequency response.

The photograph of the combined system of the acoustic filter and the electret condenser microphone is shown in Fig. 7.

\section{CONCLUSION}

Synthetic design of acoustic antialiasing filter with one resistive element is described. Computer aided method for slight modification is also introduced. Design example for an electret condenser microphone is briefly described and it shows flat amplitude-frequency characteristics below $15 \mathrm{kHz}$.

\section{ACKNOWLEDGEMENTS}

The authors were favored to have the assistance of Mr. Kazuhiko Kajihara who contributed his experimental skill, sustained effort to the development of the acoustic filter.

\section{REFERENCES}

1) J. L. Flanagan, "Acoustic filters to aid digital voice," Bell Sys. Tech. J. 58, 903-944 (1979).

2) K. Miura, M. Katakura, and S. Gotoh, "Digitalization of microphone," Preprint for the Spring Meeting, Acoust. Soc. Jpn. (1981) (in Japanese).

3) K. Kajihara and M. Katakura, "Synthetic design of acoustic filter for digitalization of condenser microphone," Preprint for the Spring Meeting, Acoust. Soc. Jpn. (1981) (in Japanese).

4) T. Sakakura, M. Kimura, and K. Miura, "New method of floating type analog to digital converter," Preprint for the Spring Meeting, Acoust. Soc. Jpn. (1981) (in Japanese).

5) E. A. Guillemin, Synthesis of Passive Networks (John Wiley \& Sons, Inc., New York, 1957), pp. 445-455. 